\title{
Multiple Dendritic Catalysts for Asymmetric Transfer Hydrogenation
}

Ying-Chun Chen, ${ }^{a}$ Tong-Fei Wu, ${ }^{a}$ Jin-Gen Deng, ${ }^{a}{ }^{*}$ Hui Liu, ${ }^{a}$ Xin Cui, ${ }^{a}$ Jin Zhu, ${ }^{a}$ Yao-Zhong Jiang, ${ }^{a}$ Michael C. K. Choi, ${ }^{b}$ and Albert S. C. Chan ${ }^{b}$

${ }^{a}$ Union Laboratory of Asymmetric Synthesis, Chengdu Institute of Organic Chemistry, the Chinese Academy of Sciences, Chengdu 610041, China

jgdeng@cioc.ac.cn

${ }^{b}$ Open Laboratory of Chirotechnology and Department of Applied Biology and Chemical Technology, The

Hong Kong Polytechnic University, Hong Kong, China

\section{Supporting Information}

\section{Table of Contents}

1. General procedure for asymmetric transfer hydrogenation of ketones

2. General procedure for asymmetric transfer hydrogenation of diones

3. General procedure for asymmetric transfer hydrogenation of imines

4. References

5. Spectra of dendritic ligands 


\section{General procedure for asymmetric transfer hydrogenation of ketones ${ }^{1}$}

$\left[\mathrm{RuCl}_{2} \text { (cymene) }\right]_{2}(1.3 \mathrm{mg}, 0.002 \mathrm{mmol})$, Dendritic ligand (1.1eq. of $\left.\mathrm{Ru}\right)$ and $\mathrm{NEt}_{3}(1.3 \mathrm{~L}$, $0.008 \mathrm{mmol}$ ) were heated in methanol at $65 \mathrm{C}$ for $2 \mathrm{~h}$, and methanol was removed under vacuum. Then ketone $\mathbf{8}(0.4 \mathrm{mmol})$ and formic acid/triethylamine azeotrope $(0.2 \mathrm{~mL})$ were added in turn. The solution was stirred at $28 \mathrm{C}$ and monitored by TLC. After completion, the solution was dilute with $3 \mathrm{~mL}$ diethyl ether, washed with brine and dried $\left(\mathrm{Na}_{2} \mathrm{SO}_{4}\right)$. The ether solution was passed through silica gel and concentrated under pressure to give $(R)$-alcohol. (Ru: ligand: substrate $=1: 1.1: 100)$

(R)-1-Phenylethanol, [ ] ${ }_{\mathrm{D}}^{28}+48.2$ (c 1.20, DCM), GC: CP-Cyclodex-236M, 110 C, $\mathrm{t}_{R}=6.9 \mathrm{~min}, \mathrm{t}_{S}=7.5 \mathrm{~min}, 96.4 \%$ ee.

(R)-1-(o-Fluorophenyl)ethanol, [ $]_{\mathrm{D}}{ }^{28}+43.2\left(\mathrm{c} 0.66, \mathrm{CHCl}_{3}\right), \mathbf{G C}$ : CP-Cyclodex-236M, $114 \mathrm{C}, \mathrm{t}_{R}=7.2 \mathrm{~min}, \mathrm{t}_{S}=7.7 \mathrm{~min}, 93.2 \%$ ee.

(R)-1-(p-Fluorophenyl)ethanol, [ $]_{\mathrm{D}}{ }^{28}+44.0\left(\mathrm{c} 0.72, \mathrm{CHCl}_{3}\right)$, GC: CP-Cyclodex-236M, $114 \mathrm{C}, \mathrm{t}_{R}=7.8 \mathrm{~min}, \mathrm{t}_{S}=8.5 \mathrm{~min}, 89.4 \%$ ee.

(R)-1-(o-Chlorophenyl)ethanol, [ $]_{\mathrm{D}}^{28}+61.0\left(\mathrm{c} 0.84, \mathrm{CHCl}_{3}\right), \mathbf{G C}: \mathrm{CP}-\mathrm{Cyclodex}-236 \mathrm{M}$, $144 \mathrm{C}, \mathrm{t}_{R}=4.9 \mathrm{~min}, \mathrm{t}_{S}=5.4 \mathrm{~min}, 95.5 \%$ ee.

(R)-1-(o-Bromophenyl)ethanol, [ ] ${ }_{\mathrm{D}}^{28}+51.7$ (c 1.36, $\left.\mathrm{CHCl}_{3}\right)$, GC: CP-Cyclodex-236M, $154 \mathrm{C}, \mathrm{t}_{R}=5.1 \mathrm{~min}, \mathrm{t}_{S}=5.7 \mathrm{~min}, 94.6 \%$ ee.

(R)-1-(p- - $^{\mathrm{t}}$ utylphenyl)ethanol, [ $]_{\mathrm{D}}{ }^{28}+44.1\left(\mathrm{c}\right.$ 0.68, $\left.\mathrm{CHCl}_{3}\right), \mathbf{G C}$ : CP-Cyclodex-236M, $120 \mathrm{C}, \mathrm{t}_{R}=12.1 \mathrm{~min}, \mathrm{t}_{S}=12.4 \mathrm{~min}, 97.2 \%$ ee.

\section{General procedure for asymmetric transfer hydrogenation of diones}

$\left[\mathrm{RuCl}_{2} \text { (cymene) }\right]_{2}(1.3 \mathrm{mg}, 0.002 \mathrm{mmol})$, Dendritic ligand (1.1eq. of $\left.\mathrm{Ru}\right)$ and $\mathrm{NEt}_{3}(1.3 \mathrm{~L}$, $0.008 \mathrm{mmol}$ ) were heated in methanol at $65 \mathrm{C}$ for $2 \mathrm{~h}$, and methanol was removed under 
vacuum. Then dione $9(0.4 \mathrm{mmol}), \mathrm{DMF}(0.2 \mathrm{~mL})$ and formic acid/triethylamine azeotrope $(0.4 \mathrm{~mL})$ were added in turn. The solution was stirred at $40 \mathrm{C}$ and monitored by TLC. After completion, the solution was dilute with $10 \mathrm{~mL}$ ethyl acetate, washed with brine and dried. Flash chromatography through silica gel (petroleum ether/EtOAc=1:3) and concentrated under vacuum to give $(R, R)$-diol. (Ru: ligand: substrate $=1: 1.1: 100)$

$(\boldsymbol{R}, \boldsymbol{R})$-1,3-Diphenyl-1,3-propanediol, ${ }^{2}[]_{\mathrm{D}}{ }^{28}+68.6\left(\mathrm{c} 1.0, \mathrm{C}_{2} \mathrm{H}_{5} \mathrm{OH}\right)$, HPLC: Chiralcel OD column, 2-propanol:hexane $=9: 91,1.0 \mathrm{~mL} / \mathrm{min}, \mathrm{t}_{S, S}=12.1 \mathrm{~min}, \mathrm{t}_{R, R}=14.2 \mathrm{~min}, \mathrm{t}_{\text {meso }}=17.5 \mathrm{~min}$, $>99 \% e e, 98.7 \%$ de.

$(\boldsymbol{R}, \boldsymbol{R})$-1,6-Diphenyl-1,6-hexanediol, ${ }^{3}[]_{\mathrm{D}}{ }^{28}+20.3\left(\mathrm{c} 0.92, \mathrm{CH}_{3} \mathrm{OH}\right)$, HPLC: ChiralPak AD column, 2-propanol:hexane $=20: 80,1.0 \mathrm{~mL} / \mathrm{min}, \mathrm{t}_{S, S}=9.0 \mathrm{~min}, \mathrm{t}_{R, R+m e s o}=10.6 \mathrm{~min}$; Chiralcel OB column, 2-propanol:hexane $=20: 80,1.0 \mathrm{~mL} / \mathrm{min}, \mathrm{t}_{S, S+m e s o}=8.4 \mathrm{~min}, \mathrm{t}_{R, R}=114.1 \mathrm{~min},>99 \%$ ee, $84.2 \%$ de.

\section{General procedure for asymmetric transfer hydrogenation of imines ${ }^{4}$}

$\left[\mathrm{RuCl}_{2} \text { (cymene) }\right]_{2}(1.3 \mathrm{mg}, 0.002 \mathrm{mmol})$, Dendritic ligand (1.1 eq. of $\left.\mathrm{Ru}\right)$ and $\mathrm{NEt}_{3}(1.3 \mathrm{~L}$, $0.008 \mathrm{mmol}$ ) were heated in methanol at $65 \mathrm{C}$ for $2 \mathrm{~h}$, and methanol was removed under vacuum. Then imine $10(0.4 \mathrm{mmol}), \mathrm{DCM}(0.8 \mathrm{~mL})$ and formic acid/triethylamine azeotrope $(0.2 \mathrm{~mL})$ were added in turn. The solution was stirred at $28 \mathrm{C}$ and monitored by TLC. After completion, the solution was dilute with $5 \mathrm{~mL}$ ethyl acetate, washed with brine and dried. Flash chromatography through silica gel (petroleum ether/EtOAc=1:4) and concentrated under vacuum to give $(R)$-amine. (Ru: ligand: substrate $=1: 1.1: 100$ )

3-Benzyl-2,3-dihydrobenzo[ $d]$ isothiazoline 1,1-dioxide, []$_{\mathrm{D}}{ }^{28}+61.5\left(\mathrm{c} 1.10, \mathrm{CHCl}_{3}\right)$, HPLC: Chiralcel OD column, 2-propanol:hexane $=20: 80,1.0 \mathrm{~mL} / \mathrm{min}, \mathrm{t}_{S}=13.2 \mathrm{~min}, \mathrm{t}_{R}=17.0$ $\min , 95.9 \%$ ee.

3-t-Butyl-2,3-dihydrobenzo[d]isothiazoline 1,1-dioxide, []$_{\mathrm{D}}^{28}+51.6\left(\mathrm{c} 0.68, \mathrm{CHCl}_{3}\right)$, HPLC: Chiralcel OD column, 2-propanol:hexane $=20: 80,1.0 \mathrm{~mL} / \mathrm{min}, \mathrm{t}_{S}=7.4 \mathrm{~min}, \mathrm{t}_{R}=17.8 \mathrm{~min}$, 
$98.3 \%$ ee.

N-t-Butylsulfonyl-1-phenylethylamine, []$_{\mathrm{D}}{ }^{28}+19.0\left(\mathrm{c} 0.85, \mathrm{CHCl}_{3}\right), \mathrm{HPLC}$ : Chiralcel AD column, 2-propanol:hexane $=10: 90,1.0 \mathrm{~mL} / \mathrm{min}, \mathrm{t}_{R}=5.71 \mathrm{~min}, \mathrm{t}_{S}=6.39 \mathrm{~min}, 51.8 \%$ ee.

\section{References}

1. (a) Hashiguchi, S.; Fujii, A.; Takehara, J.; Ikariya , T.; Noyori, R. J. Am. Chem. Soc. 1995, 117, 7562. (b) Fujii, A.; Hashiguchi, S.; Uematsu, N.; Ikariya, T.; Noyori, R. J. Am. Chem. Soc. 1996, 118, 2521. (c) Murata, K.; Ikariya, T.; Noyori, R. J. Org. Chem. 1999, 64, 2186.

2. Roos, G. H. P.; Donovan, A. R. Tetrahedron: Asymmetry 1999, 10, 991.

3. Quallich, G. J.; Keavey, K. N.; Woodall, T. M. Tetrahedron Lett. 1995, 36, 4729.

4. Ahn, K. H.; Ham, C.; Kim, S.-K.; Cho, C.-W. J. Org. Chem. 1997, 62, 7047. 
5. Spectra of dendritic ligands 


\section{Compound 9}

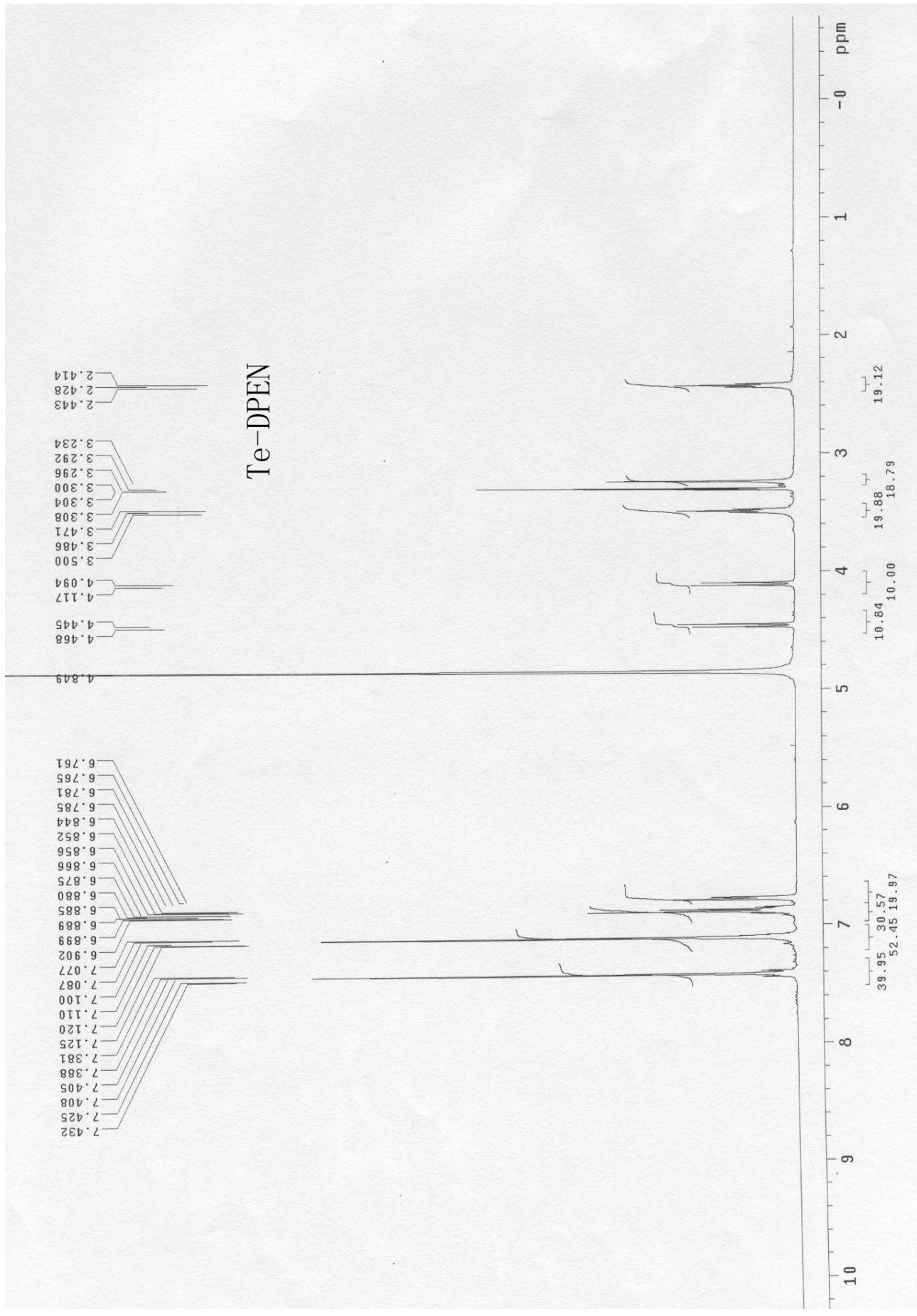




\section{Compound 9}

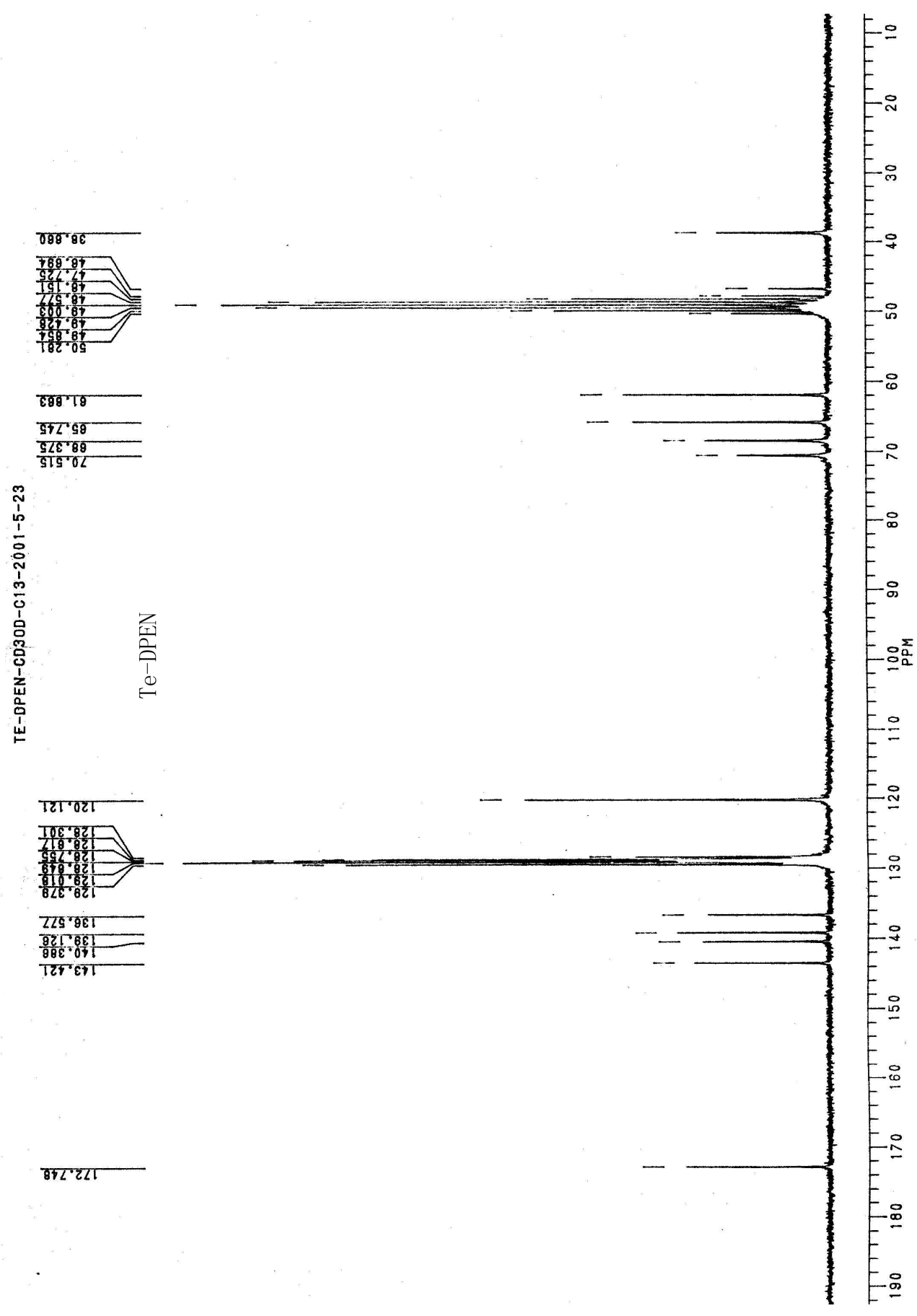


Compound 9

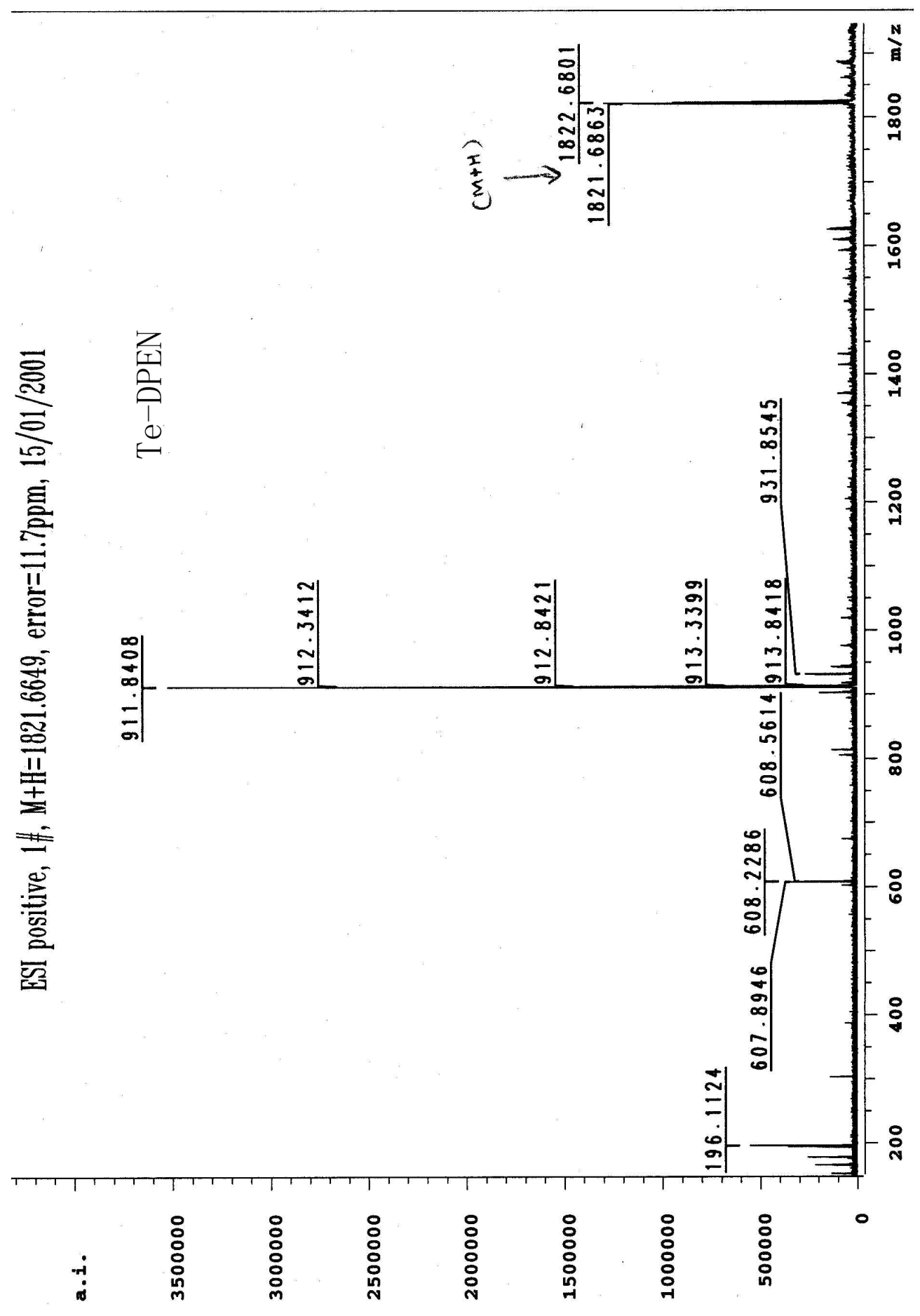




\section{Compound 10}

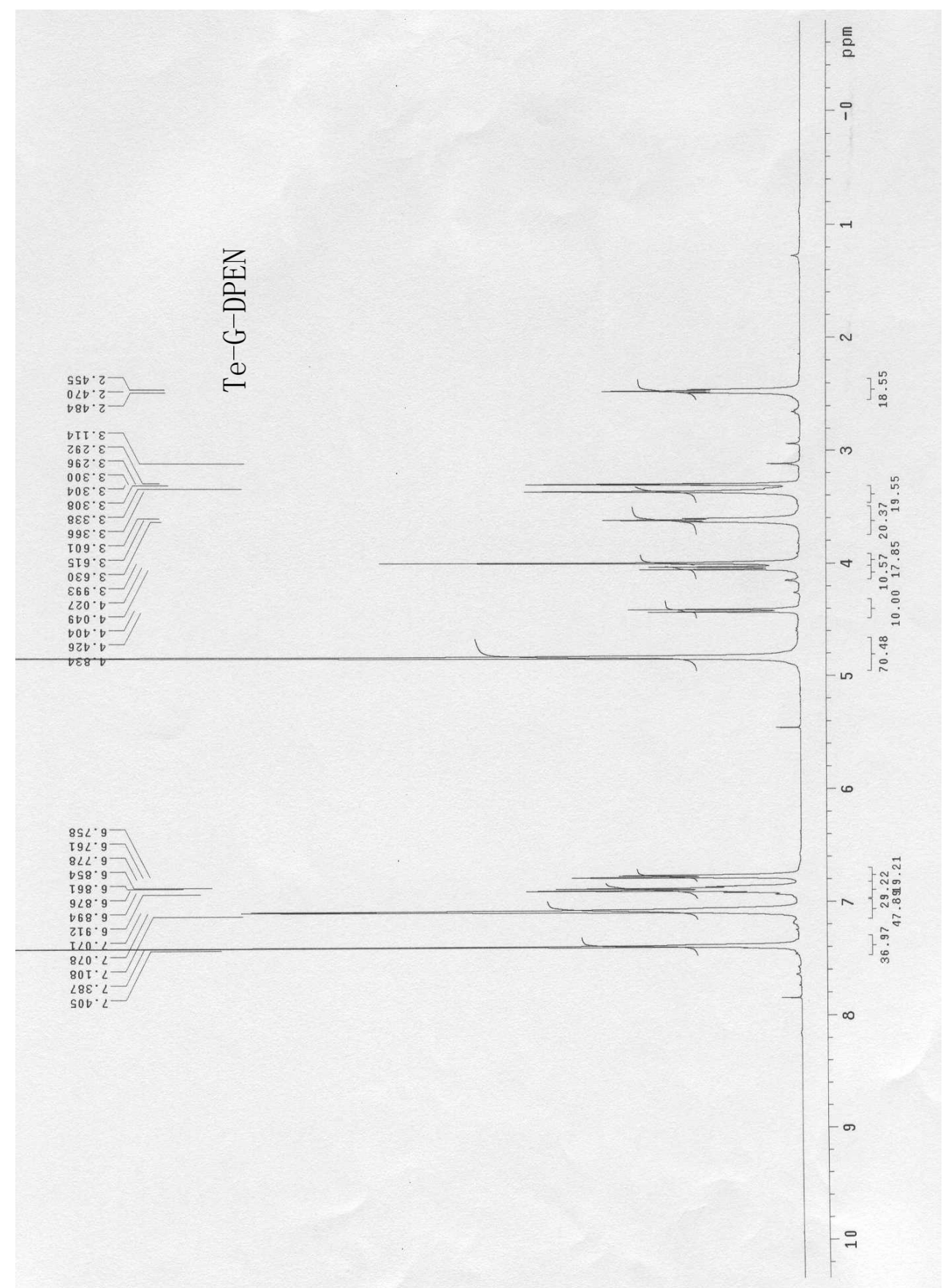




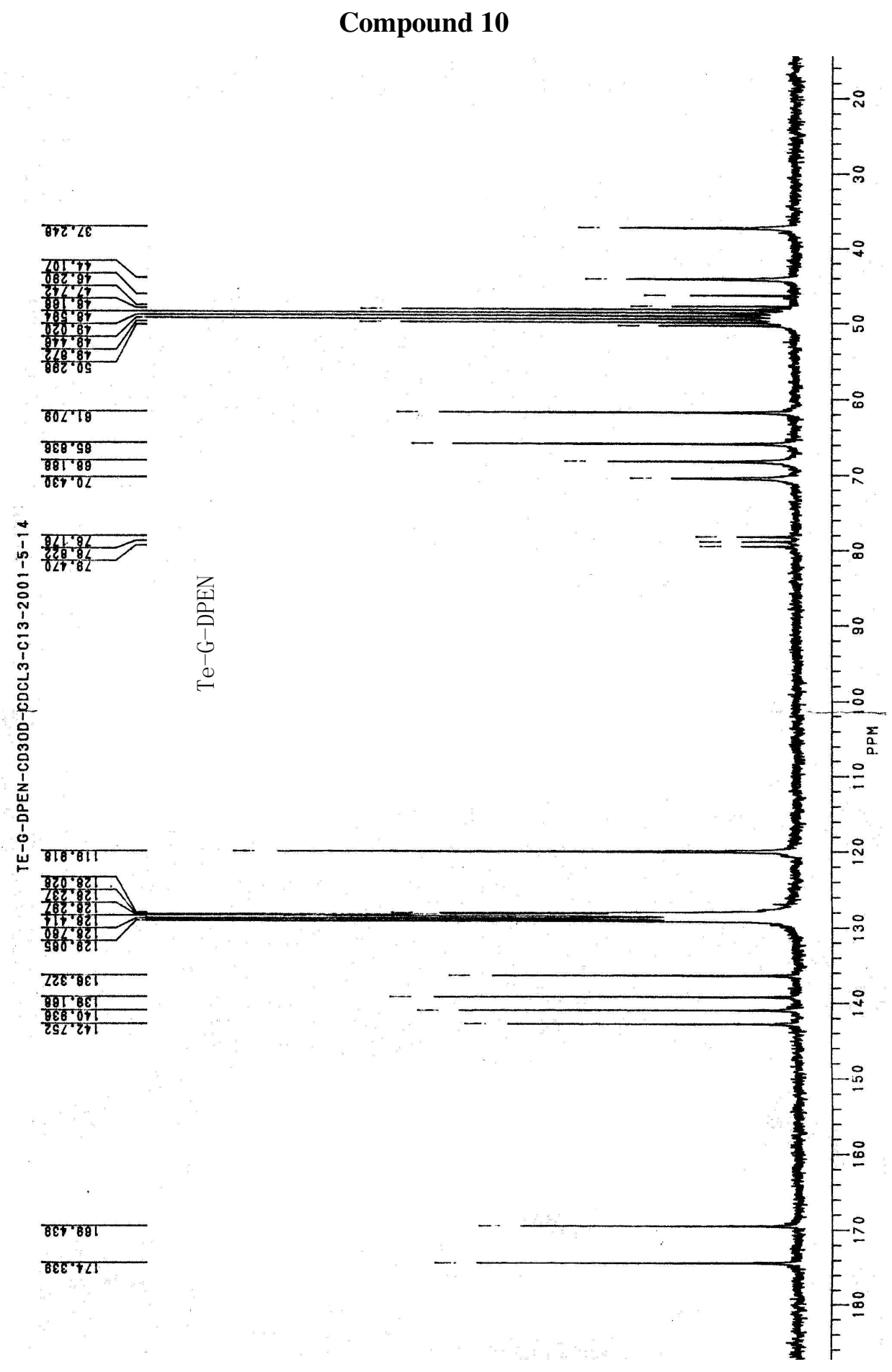




\section{Compound 10}

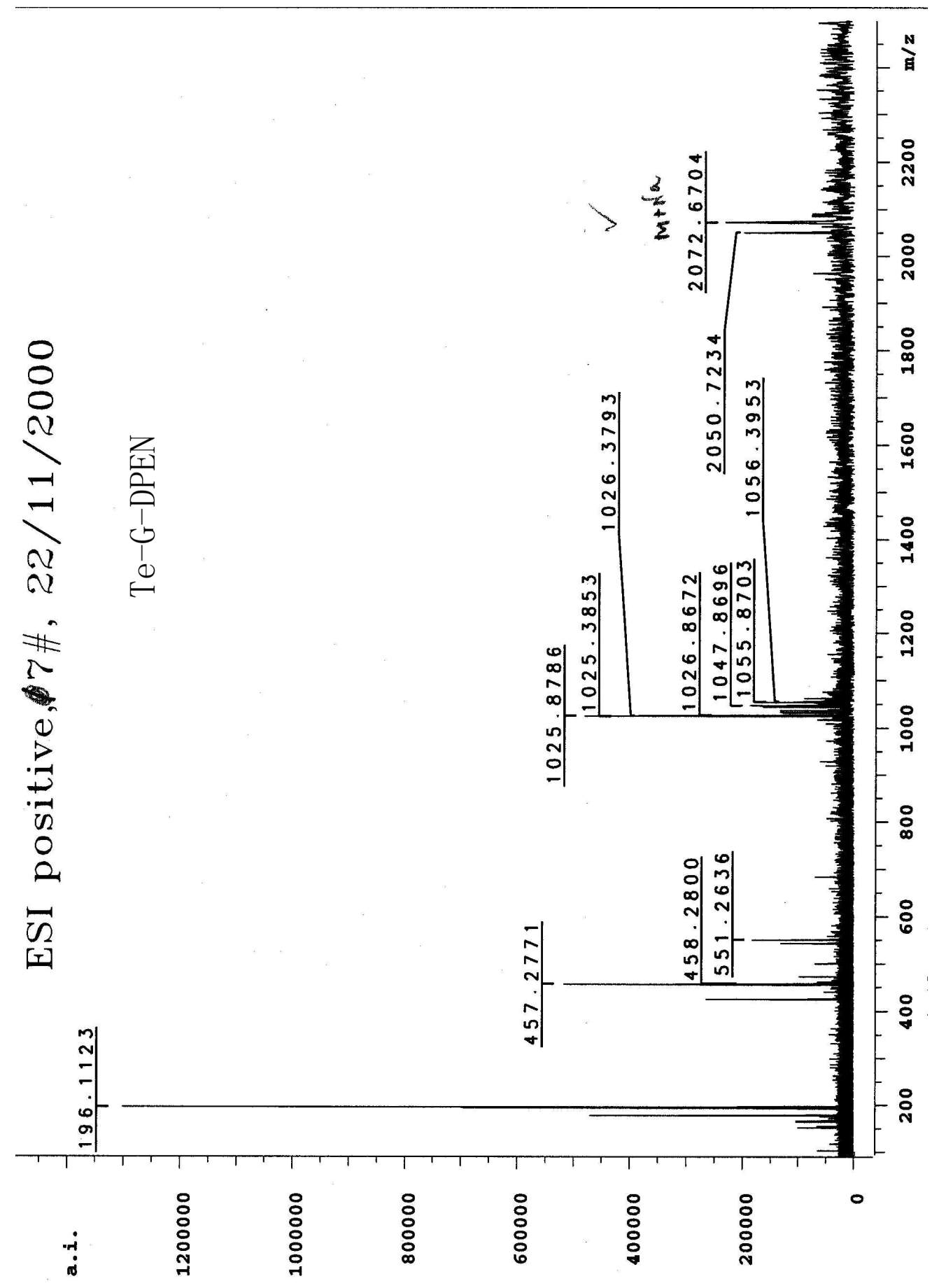




\section{Compound 16}

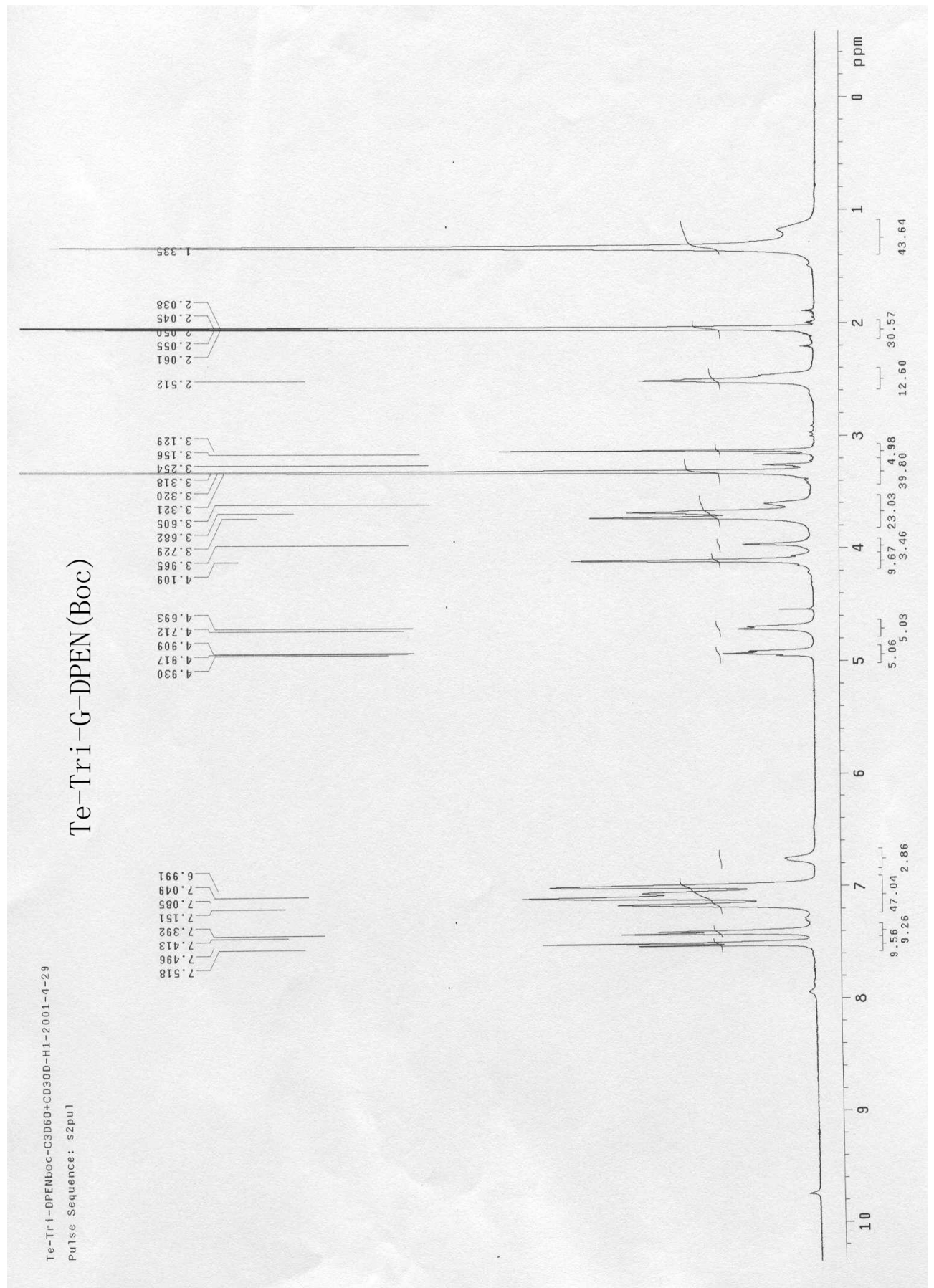




\section{Compound 17}

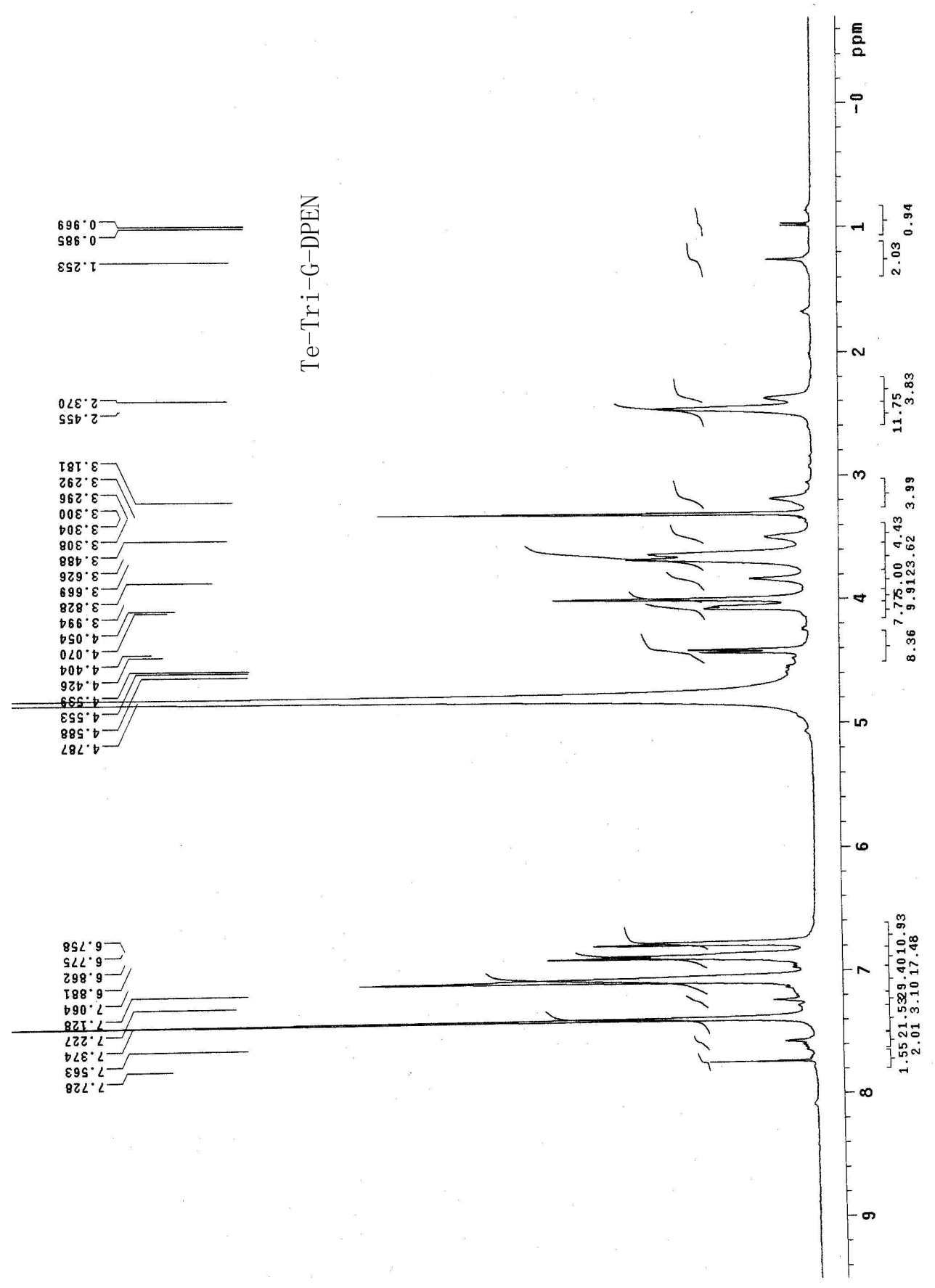




\section{Compound 17}

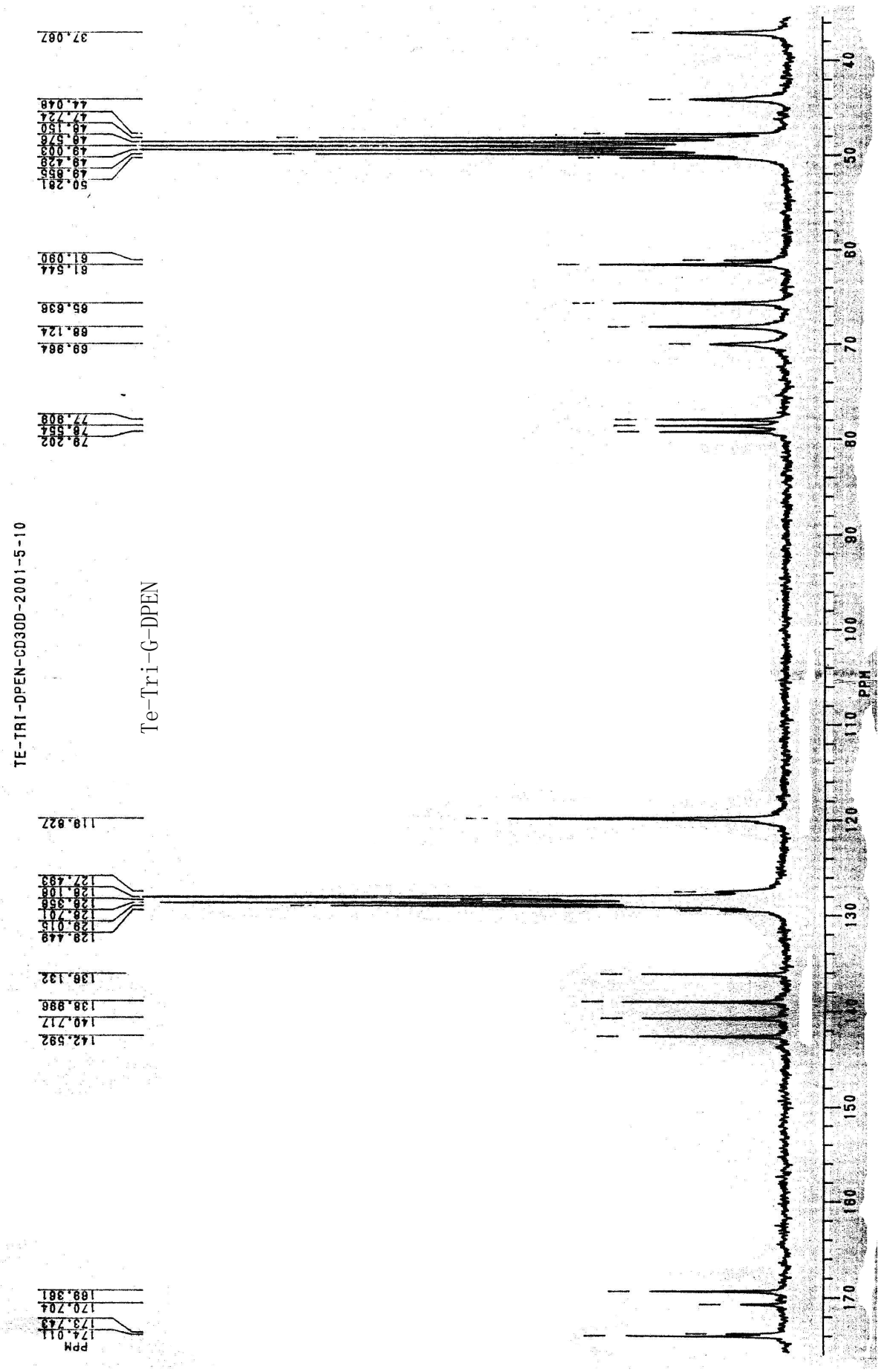




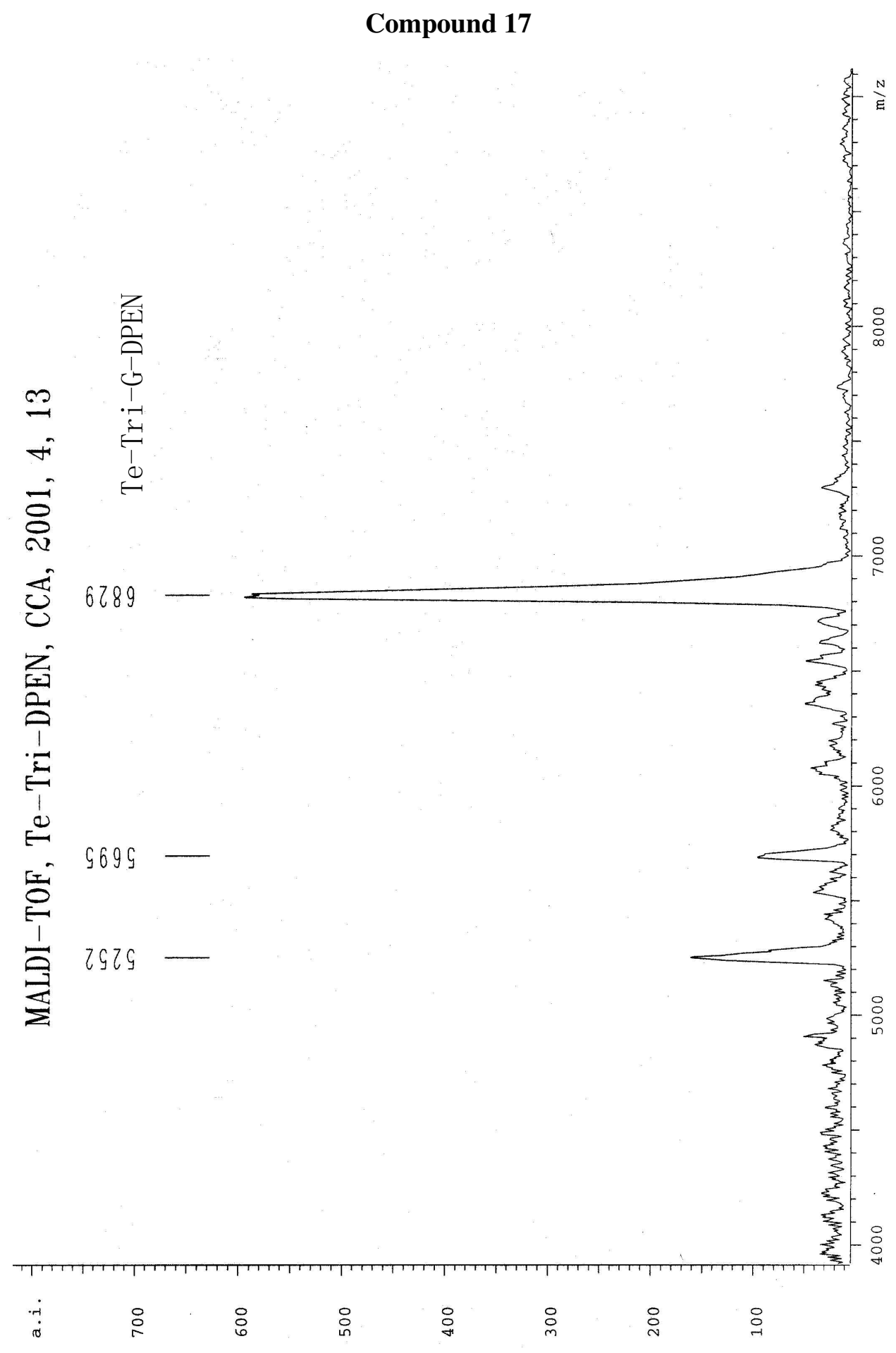

